\title{
Analysis and Design of Selection Committees: A Game Theoretic Secretary Problem LSE-CDAM-2008-03
}

\author{
Steve Alpern \\ London School of Economics, s.alpern@lse.ac.uk \\ Shmuel Gal \\ University of Haifa, sgal@univ.haifa.ac.il
}

April 9, 2008 


\begin{abstract}
Firms often delegate important decisions to committees which are set up specifically for that purpose; for example selection committees. We analyze the equilibrium behavior of a game in which committee members (the players) interview candidates sequentially, either hiring or going on to the next one. The players have differing evalutions of candidates (e.g. one cares about typing skills; the other about IT skills), which become their utilities if the candidate is hired. We then consider the optimal design (rules of the game) of such a committee, from the point of view of the firm. That is, which rules hire candidates which maximize the firm's utility.

Our committee game has a first round in which the members sequentially, by order of player number, say 'yea' or 'nea' to the candidate. If there are sufficient 'yeas' then she is tentatively hired; otherwise she is rejected. In the former case, members who said nea can veto the candidate in the second round. Thus the candidate is either hired, rejected, or vetoed. In the last case, the member casting a veto has one less to use on later candidates.
\end{abstract}

Keywords: committee, stochastic game, voting, veto 


\section{Introduction}

Often a large organization (or firm) will delegate an important decision to a committee which is specifically set up for that purpose. An important example is a selection committee designed to fill a job vacancy with the best candidate, from the firm's perspective. Committee members will have candidate evaluations (their utility, if that candidate is hired) which in general differ both from that of other members and from the firm's evaluation. The first type of difference of opinion means that the committee process comprises a game; the second type leads to a design problem (rules of the game) for the firm. As usual in such design problems, one must first analyze the equilibria in the games corresponding to various rules, and then optimize those rules.

We assume, for simplicity, that the committee decides on the candidates sequentially. Thus our model is a game theoretic extension of the well known 'secretary problem' (Ferguson (1989, 2001), Eriksson et al (2007)), though quality rather than rank of quality is optimized. For each candidate (each stage game), there are two rounds. In the first, the committee members (players) either accept or reject the candidate, voting in a fixed order (Player 1 first, etc.). If the candidate is accepted by the required number of players, she is tentatively hired; otherwise she is rejected. In the former case, any players who rejected her have the opportunity, in turn, to veto her. If any player does so, then he has one less veto available for future candidates. This process continues until a candidate is hired. Each player's utility for this event is simply his evaluation of the hired candidate, discounted (with a given discount factor $d$ ) by the number of candidates who have been considered (number of periods). A related problem in which two committee members need to fill two positions (one candidate working for each member) has been analyzed by Baston and Garnaev (2007).

We realize that it is unusual for actual committee members to be given a fixed number of vetoes, but this assumption should be viewed as a formalization of the recognized fact that pressure towards consensus prevents members from continuing to veto successive candidates, and that this pres- 
sure increases as they veto more and more candidates. Often committees in fact require consensus to make appointments; after an initial round in which a single member is against the candidate, there may be a discussion in which the others attempt to get him to change his mind - if he refuses, this can be interpreted as a veto. In some cases, for example jury selection (see Brams and Davis (1976, 1978) and DeGroot and Kadane (1980)), fixed numbers of vetos are indeed specified explicitly.

We analyze the case where the committee consists of two members. Thus each candidate has an evaluation pair $z=(x, y)$ (observable to both players) which we can normalize to the unit square. Thus $x$ measures say the candidates typing skill, which is what matters to Player I, while $y$ measures her IT skill which corresponds to Player II's utility. We mainly assume that the distribution of candidates is the uniform distribution $\bar{\mu}$ over the square, but we have some general results for an arbitrary or symmetric distribution $\mu$. We denote the game where Player I starts with $i$ vetoes and Player II starts with $j$ vetoes as $G(i, j)$. While the no-veto game $G(0,0)$ is reasonably straightforward, as soon as there are vetos available the actions of the players are more strategic. For example a player may accept a candidate he would prefer not to be hired if he knows the other player thinks she is very bad and will be forced to use up a veto. The timing of moves is important in this respect, and our equilibrium notion includes the element of subgame perfectness.

For the uniform candidate distribution $\bar{\mu}$, it turns out (Theorem 17) that there is a unique equilibrium. Thus for any $d$, the firm can determine the number of vetos each player should start with in order to maximizes it's own utility function of $x$ and $y$. For example, we find that when the firm is neutral between the players' utilities, valuing candidates as $x+y$, the optimal number of vetos for $d=.91$ is two for Player I andthree for Player II. The differing numbers of vetos reflects the fact that the game is not symmetric between the players (even if $i=j$ ) because Player I has the advantage of going first in round 1 of each stage game.

A general finding is that it is always better to have more vetos. For the uniform candidate distribution, where equilibria are unique, it is always 
better for a player if the other player has fewer vetos.

\section{The Game $G(i, j)$ and its Equilibria}

The game $G(i, j)$ is easy to describe formally. The first move is a chance move in which a candidate type $z=(x, y)$ is drawn from the distribution $\mu$, independently of previous choices. Then players I and II in turn accept (a) or reject (r) the candidate. If both accept, the candidate is hired and the payoff vector is $(x, y)$. If both reject, the candidate is rejected and the game $G(i, j)$ is repeated (with payoffs discounted by the factor $d$ ). If the players disagree, the player who rejected can either veto (v) (if he has one left) and play the game again with one less veto; or not veto (n), in which case the candidate is hired, with payoff vector $(x, y)$. This game tree is drawn below in Figure 1. If one or more players has no vetos ( $i$ or $j$ is 0 ) then the second stage is removed and replaced by the terminal node with payoff $(x, y)$. (Alternatively, a 'virtual' veto may be allowed, resulting in a degenerate game say $G(-1, j)$ which has constant payoff 0 for Player I so it will never be used.)

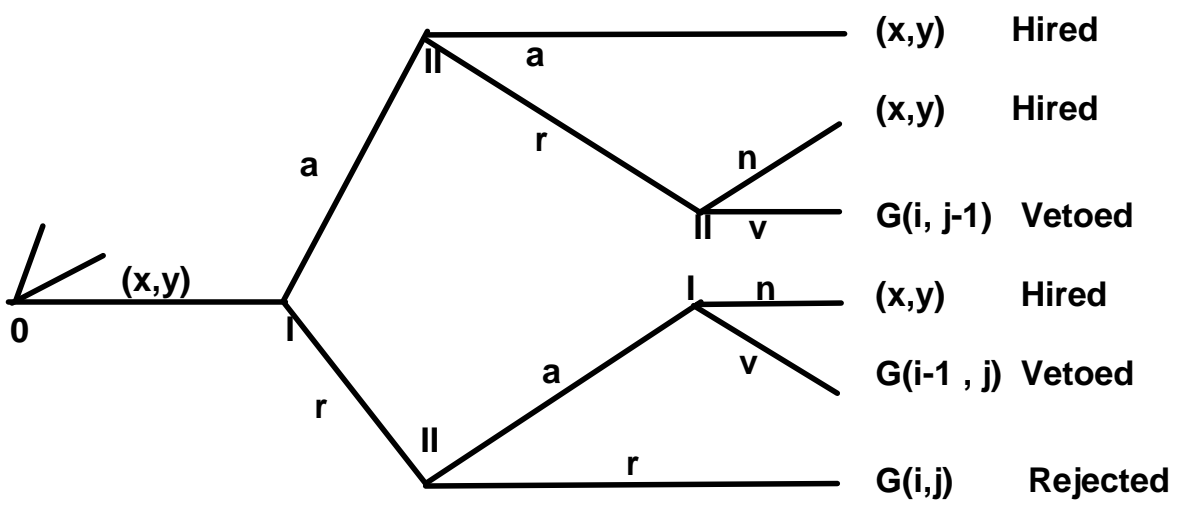

Figure 1: The game tree for $G(i, j)$. 


\subsection{Strategies and equilibria}

A strategy for a player is simply a rule as to when to accept or reject a candidate in the first round; and if rejecting, whether to veto in the second round. The rule depends on the candidate values $(x, y)$ and on all the moves in the stage game, but not on moves of the players in earlier stages, if any. In the event that both players reject a candidate, the same stage game $G(i, j)$ will be repeated and in this case we assume the players use the same strategies - a stationarity assumption. Given this stationarity condition, our equilibrium concept is subgame perfect Nash. We make this explicit in the following.

Definition 1 (equilibrium for $G(i, j)$ ) Suppose we fix equilibria in the subgames $G(i-1, j)$ and $G(i, j-1)$ with respective values (expected payoffs) $\left(U_{-}, V_{+}\right)$in $G(i-1, j)$ and $\left(U_{+}, V_{-}\right)$in $G(i, j-1)$. A strategy for the stage game $G(i, j)$ is an equilibrium supported by these if it has respective values (expected payoffs) of $(U, V)$ and for every candidate $(x, y)$ it is a subgame perfect solution to the extensive form game drawn below in Figure 2.

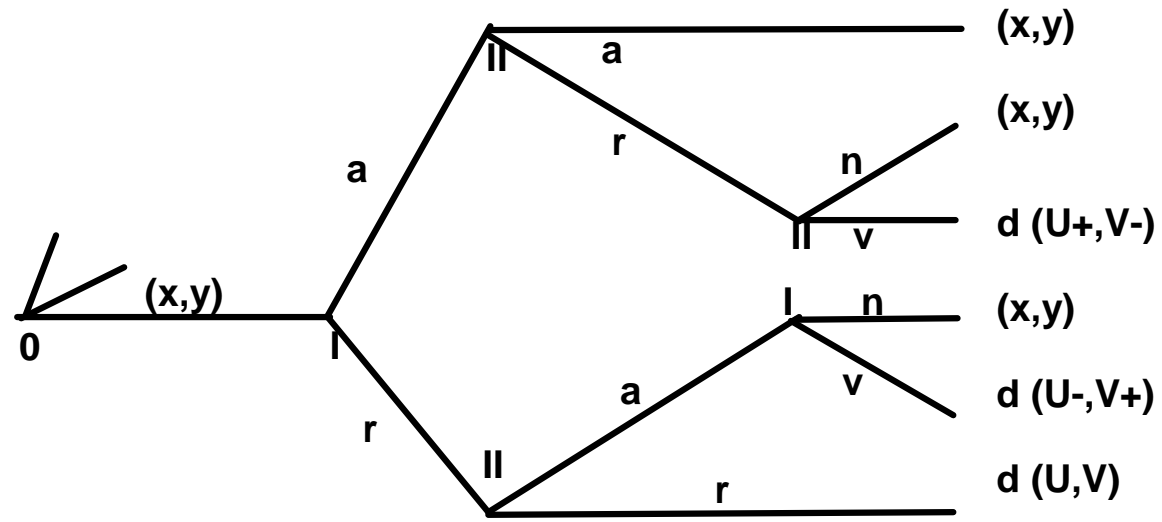

Figure 2: Tree for $G(i, j)$ with payoffs.

Note that for this recursive definition we have $U_{-}=0$ if $i=0$ and $V_{-}=0$ if $j=0$, which is equivalent to removing the veto option in these cases. An 
easy observation is that in the undiscounted game it can never hurt a player to have an extra veto, as stated more precisely in the following result which justifies the notation $U_{-}$as something less than $U$.

Lemma 2 Let $(U, V)$ be the value of an equilibrium strategy in the undiscounted game $G(i, j)$ which is supported by equilibria in the subgames $G(i-1, j)$ and $G(i, j-1)$ with respective values $\left(U_{-}, V_{+}\right)$and $\left(U_{+}, V_{-}\right)$. Then

$$
U \geq U_{-} \text {and } V \geq V_{-}
$$

Proof. Consider the game tree of Figure 2 for the case where $x<U_{-}$. If Player I chooses $r$ as his first move then the candidate $(x, y)$ will not be hired, as he will veto if necessary in the second round. So $r$ for I leads to a payoff for I of either $U$ or $U_{-}$. In the case $x \geq U_{-}, r$ also cannot lead to a terminal value for I of less than $\min \left(U, U_{-}\right)$. Consequently the expected value for I of $U$ must satisfy $U \geq \min \left(U, U_{-}\right)$. But this expected value is by definition $U$, so $U \geq \min \left(U, U_{-}\right) \geq U_{-}$. The argument for the other inequality is similar (but not symmetric, as the game is not symmetric): Suppose $y<V_{-}$. Then if I accepts, II will reject and veto, getting $V_{-}$. If I rejects, then II will not get less than $V_{-}$(obtainable by also rejecting). Hence II gets at least $\min \left(V, V_{-}\right)$. The same is true more easily if $y \geq V_{-}$and so the for any equilibrium the expected value $V$ for II is at least $\min \left(V, V_{-}\right)$, so $V \geq V_{-}$.

\subsection{Equilibria in $G(i, j)$ with vetos}

We now describe the nature of equilibria in $G(i, j)$ for general $d<1$ and arbitrary $i$ and $j$. For the uniform candidate distribution $\bar{\mu}$ it will turn out that equilibria are unique and it is always better for a player to have more vetoes and for his opponent (other player) to have fewer vetoes. In the notation of Lemma 2, we say that an equilibrium is progressive if its values $(U, V)$, compared with $\left(U_{-}, V_{+}\right)$and $\left(U_{+}, V_{-}\right)$of its two supporting equilibria, satisfy

$$
U_{-}<U<U_{+} \text {and } V_{-}<V<V_{+} \text {. }
$$


To simplify our notation, we denote the discounted versions of these values by lower case letters, as

$$
u_{-}=d U_{-}, u=d U, u_{+}=d U_{+}, v_{-}=d V_{-}, v_{+}=d V_{+} .
$$

$u, v$ represent threshold values, but at equilibrium, $u=d U, v=d V$

In the final round, Player I would veto any candidate with $x<u_{-}=d U_{-}$. Similarly, he would prefer to hire any candidate with $x \geq u=d U$, since the latter is what he would get if the game were repeated. According to our progressive assumption (1), each player would prefer the other to lose a veto rather than repeat the current game. So for example in solving the game tree of Figure 2, Player I would accept any candidate $(x, y)$ with $y<v_{-}=d V_{-}$ (the rectangle $A$ in Figure 3 ), even one with $x=0$ ! Progressive equilibria must have the form depicted in Figure 3 (with $a$ for accept, $r$ for reject but not veto, $v$ for reject and veto if necessary).

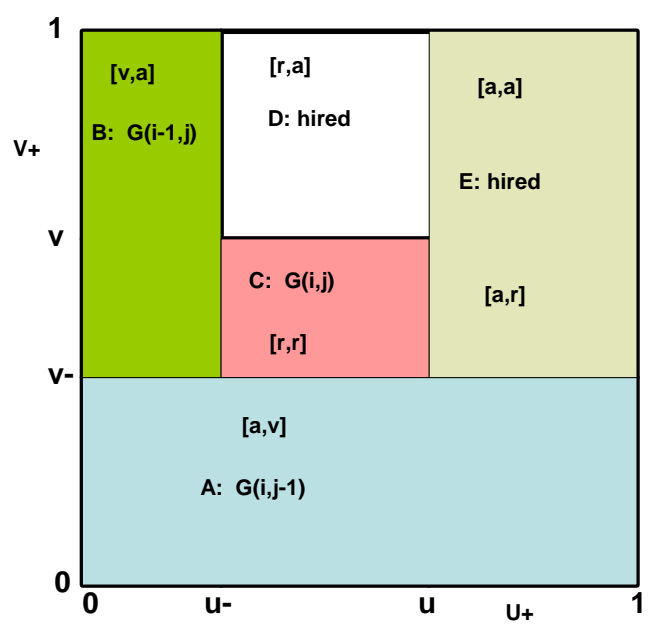

Figure 3. The strategy $[u, v]_{\text {prog }}$.

The expected payoff vector corresponding to the strategy $[u, v]_{\text {prog }}$, denoted 
by $T(u, v)$, is given by

$$
\begin{gathered}
T(u, v)=\mu(A) \cdot\left(u_{+}, v_{-}\right)+\mu(B) \cdot\left(u_{-}, v_{+}\right)+\mu(C) \cdot(u, v) \\
+\int_{D \cup E}(X(z), Y(z)) d \mu(z),
\end{gathered}
$$

where $X$ and $Y$ are the projections $X(z)=x$ and $Y(z)=y$ if $z=(x, y)$. We view the arguments of $T$ as threshold acceptance levels, rather than values. If the measure $\mu$ has full support (and $d=1$ ), then the equilibrium condition is simply $T(u, v)=(U, V)=(u, v)$. If $\mu$ does not necessarily have full support and $\mu\left(\left[u^{\prime}, u\right) \times\left[v^{\prime}, v\right)\right)=0$, then the strategies $\left[u^{\prime}, v^{\prime}\right]_{\text {prog }}$ and $[u, v]_{\text {prog }}$ are equivalent in the sense that they have the same effect on all candidates, and we write $\left[u^{\prime}, v^{\prime}\right]_{\text {prog }} \sim[u, v]_{\text {prog }}$. Summarizing this analysis, we can represent progressive equilibria as the set of solutions to a fixed point problem.

Theorem 3 Let $\left(U_{+}, V_{-}\right)$and $\left(U_{-}, V_{+}\right)$be equilibrium values of $G(i, j-1)$ and $G(i-1, j)$. Then a strategy profile $[u, v]_{\text {prog }}$ (as described in Figure 3) with $(u, v) \in\left[u_{-}, u_{+}\right] \times\left[v_{-}, v_{+}\right]$is a progressive equilibrium for $G(i, j)$ with candidate distribution $\mu$ if and only if it satisfies the fixed point equation

$$
(u, v) \sim d \cdot T(u, v)
$$

where $T$ is given in terms of $\left(u_{+}, v_{-}\right),\left(u_{-}, v_{+}\right)$, and $\mu$ in (3). In this case the equilibrium payoffs are $U=u / d$ and $V=v / d$.

Example 4 (Uniform Distribution) If $\mu$ is simply area (Lebesgue measure) on the square, the expected value mapping $T$ can be easily calculated as the sum of the area of each rectangle multiplied by its expected payoff vector (center of gravity).

$\begin{array}{cccccc}\text { rectangle } & A & B & C & D & E \\ \text { probability } & v_{-} & u_{-}\left(1-v_{-}\right) & \left(v-v_{-}\right)\left(u-u_{-}\right) & \left(u-u_{-}\right)(1-v) & (1-u)\left(1-v_{-}\right) \\ \text {utility to } I & u_{+} & u_{-} & u & \left(u+u_{-}\right) / 2 & (1+u) / 2 \\ \text { utility to } I I & v_{-} & v_{+} & v & (1+v) / 2 & \left(1+v_{-}\right) / 2\end{array}$


Consequently $T=\left(U=T_{1}, V=T_{2}\right)$ is given by

$$
\begin{aligned}
U= & u / d=v_{-} u_{+}+u_{-}\left(1-v_{-}\right) u_{-}+\left(v-v_{-}\right)\left(u-u_{-}\right) u \\
& +\left(u-u_{-}\right)(1-v)\left(u+u_{-}\right) / 2+(1-u)\left(1-v_{-}\right) \frac{1+u}{2} \\
V= & v / d=v_{-} v_{-}+u_{-}\left(1-v_{-}\right) v_{+}+\left(v-v_{-}\right)\left(u-u_{-}\right) v \\
& +\left(u-u_{-}\right)(1-v)(1+v) / 2+(1-u)\left(1-v_{-}\right) \frac{1+v_{-}}{2}
\end{aligned}
$$

Example $5(x+y=1$ (jury selection)) Assume that all candidates lie on the line $x+y=1$, so that the evaluations of the two Players are opposite. One interpretation is that the candidate is to decide on an issue where the Players take opposite sides, and $x$ is the probability he will vote for Player I's favoured option. This is essentially a problem of selecting a jury of size 1. If $d=1$ (no discounting) then this is a constant sum game, so that there exist unique equilibrium values $U_{i, j}$ and $V_{i, j}$. In fact this remains true for any discount factor $d$, because the double rejection possibility never occurs, and the equilibrium equations can be solved for $U_{i, j}$ and $V_{i, j}$ by simple backwards induction. To see this, first observe that (for any $d, i, j$ ) we have $U_{i, j}+V_{i, j} \leq 1$, since for any candidate selected in period $n$ we have total payoff

$$
d^{n}(x+y) \leq x+y=1 \text {. }
$$

Consequently $u_{i, j}+v_{i, j}=d\left(U_{i, j}+V_{i, j}\right) \leq 1$. So any candidate $(x, y)$ satisfies either $x \geq u_{i, j}$ or $y \geq v_{i, j}$ and will be accepted (only) by the corresponding player. Thus the cases (rectangles) $C$ and $E$ will be omitted from the expected value formula (3). In particular, any candidate not vetoed by one of the players (i.e. with $x \geq u_{i-1, j}$ or $x \leq 1-v_{i, j-1}$ with $y \geq v_{i, j-1}$ ) will be accepted by one of the players. For the uniform distribution on the line $x+y=1$, the equilibrium equations for $u=u_{i, j}$ and $v=v_{i, j}$ are obtained as,

$(u, v) / d=u_{-}\left(u_{-}, v_{+}\right)+\left(1-v_{-}-u_{-}\right)\left(\frac{1+u_{-}-v_{-}}{2}, \frac{1-u_{-}+v_{-}}{2}\right)+\left(v_{-}\right)\left(u_{+}, v_{-}\right)$.

The important thing to note is that, unlike our general equilibrium equations, 
the unknowns $u$ and $v$ do not appear on the right hand side. For the case $i=j=0$ we may also use this equation with $u_{-}=v_{-}=0$ (if a player uses a veto he does not have, he gets 0$)$ giving

$$
U=V=\frac{1}{2} \text {, and } u=v=\frac{d}{2} .
$$

Thus every candidate with $x \geq d / 2$ is accepted by I and every candidate with $x \leq 1-d / 2$ is accepted by II, and the game ends in the initial period. The full jury selection game, that of selecting a many-person jury to optimize utilities of the Prosecution or the Defence (e.g. the probability of a successful verdict) has been analyzed in the classic papers of Brams and Davis (1976,1978), Roth, Kadane and DeGroot (1977) and DeGroot and Kadane (1980), among others.

\section{Equilibria in $G(0,0)$}

What do equilibrium strategy pairs look like in $G(0,0)$ ? First consider $d=1$. Suppose there is an equilibrium with values $(U, V)$. Clearly Player I should accept any candidate $(x, y)$ with $x>d U$ and reject any with $x<d U$. Either is possible for $x=d U$, but for simplicity we will assume such a candidate is accepted. Similarly Player II accepts iff $y \geq d V$. Let $(u, v)$ denote the strategy pair where I accepts $x \geq u$ and II accepts $y \geq v$, which

will be an equilibrium if $u=d U$ and $v=d V$. The strategy $(u, v)$ will hire candidates in the set

$$
H=H(u, v)=\{(x, y): x \geq u \text { or } y \geq v\}
$$




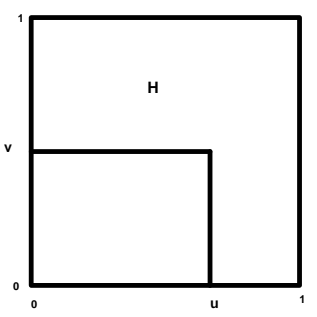

Figure 4. $H(u, v)$.

The calculation of the expected payoff pair $T(u, v)$ corresponding to $\langle u, v\rangle$ is a straightforward simplification of (3). If the candidate is hired in period $k=0,1, \ldots$, the Player I will get a mean payoff of $E_{1}(H) \cdot d^{k}$, where

$$
E_{i}(S) \text { is the mean value of the } i \text { 'th coordinate on } S \text {. }
$$

Let $p=\mu(H)$. Then the probability that the candidate is hired in period $k$ is $(1-p)^{k} p$, so

$$
\begin{aligned}
T_{i}(u, v) & =\Sigma_{k=0}^{\infty}(1-p)^{k} p \cdot E_{i}(H) d^{k} \\
& =p \cdot E_{i}(H) \Sigma_{k=0}^{\infty}((1-p) d)^{k} \\
& =\frac{p \cdot E_{i}(H)}{1-(1-p) d}
\end{aligned}
$$

The strategy pair $(u, v)$ is an equilibrium if it satisfies the fixed point (equilibrium) equation $(U, V)=T(u, v)$, or

$$
(u, v)=d T(u, v)
$$

and the equilibrium values will be $U=u / d$ and $V=v / d$.

An alternative derivation of this equation is to evaluate the expected value function $T$ as follows. If $(x, y) \notin H$, then Player I gets the value $u=d U$ next period, so he gets $d(u / d)=u$. If $(x, y) \in H$, then he gets 
$E_{1}(H)$. Thus we have at equilibrium

$$
\begin{aligned}
T(u, v) & =(1-p)(u, v)+p\left(E_{1}(H), E_{2}(H)\right), \text { and } \\
d T(u, v) & =(u, v) \text { or } \\
(u, v) & =d\left((1-p)(u, v)+p\left(E_{1}(H), E_{2}(H)\right)\right),
\end{aligned}
$$

which is the same as (7)

We illustrate the equilibrium concept in $G(0,0)$ for three special distributions, the uniform distribution over the square, an i.i.d. distribution with two atoms, and a family of i.i.d. distributions with three atoms.

Example 6 ( $G(0,0)$ with the uniform distribution) For the uniform distribution, $\mu(H)=p=1-u v, E_{1}(H)=\frac{1-u^{2} v}{2(1-u v)}$, and $E_{2}(H)=\frac{1-v^{2} u}{2(1-u v)}$, so

$$
T(u, v)=\frac{1-u v}{1-u v d}\left(\frac{1-u^{2} v}{2(1-u v)}, \frac{1-v^{2} u}{2(1-u v)}\right) .
$$

For $d=1$ the solution to the undiscounted equilibrium equation $(u, v)=$ $T(u, v)$ is

$$
u=v=\frac{\sqrt{5}-1}{2} \approx 0.61803, \text { the 'golden mean'. }
$$

The two equilibrium equations of (11) are pictured below in Figure 5. The intersection at $u=v=1$ does not represent an equilibrium because for the strategy $u=v=1$ no candidates will be hired - the formula for $T$ is only valid for $u$ and $v$ less than 1 in the undiscounted case. (With discounting $T(1,1)=0$.

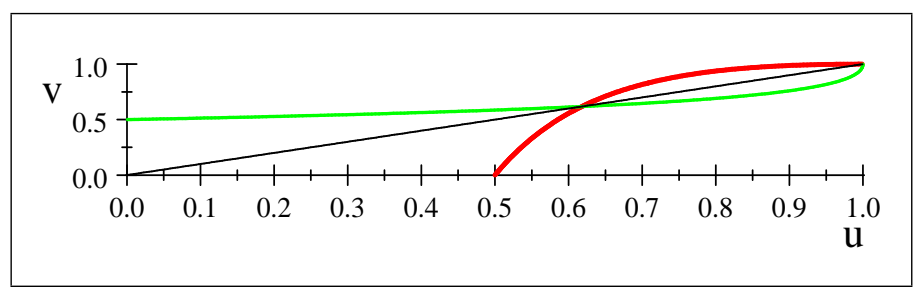

Figure 5. Equilibrium equations for u (thick red) and $v$ (thin green).

The phantom equilibrium disappears when discounting is introduced, as il- 
lustrated below for $d=.8$ in Figure 6 , where we plot the two coordinates of the general equilibrium equation $(u, v)=d T(u, v)$. The reader should also note for future reference that the equilibrium is the symmetric point

$(0.43232,0.43232)$.

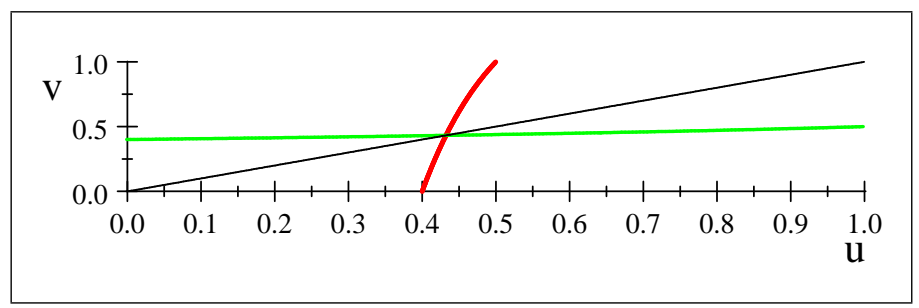

Figure 6. Equilbrium with discount factor $d=.8$.

Example 7 (i.i.d. distribution with two atoms, undiscounted) Suppose $x$ and $y$ have the distribution $F$, which has atoms at $a<b$, probability $p$ at a. Let $B=(b, b)$ and $A=(a, a)$ be the choosy and easy strategy profiles. If either player (or both) adopts cutoff value a, the game ends in the first period and both player' payoff is the mean $m$ of $F$,

$$
m=p a+(1-p) b
$$

If both adopt b, they both get

$$
T_{1}(b, b)=T_{2}(b, b)=\frac{p(1-p)+a(1-p) b}{1-p^{2}}=m^{+}, a<m<m^{+}<b,
$$

a weighted average of $a$ and $b$ more heavily weighted to $b$. Hence the payoff matrix is

\begin{tabular}{l|l|l|} 
& $a$ & $b$ \\
\hline$a$ & $(m, m)$ & $(m, m)$ \\
\hline$b$ & $(m, m)$ & $\left(m^{+}, m^{+}\right)$ \\
\hline
\end{tabular}

Hence there are two Nash. equilibria, $A=(a, a)$ and $B=(b, b)$. The (flat) easy equilibrium $A$ does not satisfy our equilibrium notion (Definition 1) because it is not subgame perfect, as $u=a<m$. To be specific, suppose the candidate is $(a, a)$ and I rejects. Then II should reject because getting $m$ on 
average next period is better than getting $a<m$ now. Given this behavior of II, I should reject candidate $(a, a)$, knowing that II will also reject and both will get $m$ next period. Thus the equilibrium condition for say Player I is that

$$
\begin{aligned}
u & \sim T_{1}(u, v)=T_{1}(u, u) \text { (for symmetric equilibrium), where } \\
w & \sim z \Longleftrightarrow \operatorname{Pr}[x \geq w]=\operatorname{Pr}[x \geq z] .
\end{aligned}
$$

Thus at the Nash equilibrium $A=(a, a)$, we have

$$
\begin{aligned}
u & =a \nsim T_{1}(a, a)=m, \text { as } \\
\operatorname{Pr}[x & \geq a]=1>\operatorname{Pr}[x>m]=\operatorname{Pr}[x=b]=1-p .
\end{aligned}
$$

So this is not a subgame perfect equilibrium. However at $(b, b)=B$, we have

$$
\begin{aligned}
u & =b \sim T_{1}(b, b)=m^{+}, \text {because } \\
\operatorname{Pr}[x & \geq b]=\operatorname{Pr}[x=b]=\operatorname{Pr}\left[x>m^{+}\right] .
\end{aligned}
$$

So in the sense of Definition 1, there is a unique equilibrium.

\section{Symmetry of Equilibria $G(0,0)$}

Recall from Example 6 that for the uniform distribution, the only equilibrium that we found was symmetric; both players used the same threshold acceptance level. Similarly, for the two atom i.i.d. distribution of Example 7 , the only equilibrium was symmetric. In this section we will show that this is true for $G(0,0)$ whenever the distribution $\mu$ is symmetric, e.g. with $x$ and $y$ i.i.d. Note that the no-veto game $G(0,0)$ is the only one which is symmetric with respect to the players, as the order of play is irrelevant. It is equivalent to the game where the players move (accept or reject the candidate) simultaneously. Such games typically have symmetric equilibria, but often have asymmetric equilibria as well. We show that this is not the case for $G(0,0)$. 
We first show that the choosy player (the one with a higher acceptance threshold) always does worse than the other player. We phrase this for I being more choosy.

Lemma 8 If $v<u$ and $F(v)<F(u)$, then $T_{2}(u, v)>T_{1}(u, v)$.

Proof. If strategies $u$ and $v$ are played, candidates will be accepted if they are in $H=H(u, v)=W \cup S$ (in Figure 7). Candidates in $S$ have the same expected values for $x$ and $y$, since $S$ is symmetric. That is, $E_{1}(S)=$ $E_{2}(S)$. However on $W, x<y$, so $E_{1}(W)<E_{2}(W)$. So assuming that the probability of the set $W$ is positive (that is, $F(v)<F(u)$ ) we have $T_{2}(u, v)=E_{2}(H)>E_{1}(H)=T_{1}(u, v)$, as claimed.

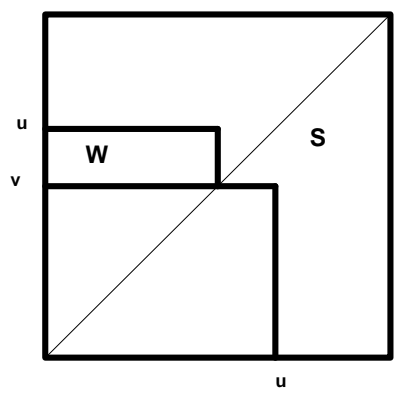

Figure 7. Choosy player does worse.

Theorem 9 If the candidate distribution is symmetric (in particular, if $x$ and $y$ are i.i.d.), then $G(0,0)$ has no asymmetric equilibria.

Proof. Suppose $[u, v]$ is a equilibrium for $G(0,0)$ with $v<u$. If it is an equilibrium, then

$$
\begin{aligned}
& T_{1}(u, v)=u, \text { and } \\
& T_{2}(u, v)=v .
\end{aligned}
$$


By the lemma, we have $T_{2}(u, v)>T_{1}(u, v)$. Hence

$$
\begin{aligned}
& u=T_{1}(u, v)<T_{2}(u, v)=v, \text { or } \\
& u<v \text {, contrary to assumption. }
\end{aligned}
$$

As an application of this result, we may simplify the analysis given in Example 6, determining the equilibrium for the uniform distribution by considering only symmetric equilibria, that is, solutions to the single equation $u=d T_{1}(u, u)$. Using (11), we write

$$
\begin{aligned}
& u=d T(u, u)=d \frac{1-u^{2}}{1-d u^{2}} \frac{1-u^{3}}{2\left(1-u^{2}\right)}, \text { with solution } \\
& d=\frac{2 u}{u^{3}+1}, \text { whose inverse we write as } u=g(d) .
\end{aligned}
$$

Example 10 (i.i.d. distribution with three atoms, undiscounted) Theorem 9 considerably simplifies the equilibrium analysis of i.i.d. distributions, by restricting the search to symmetric strategies. Assume that $F$ has atoms at $0,1 / 2,1$, with probabilities $r, p, q$. The only symmetric strategies, up to equivalence, have $u=v \in\{0,1 / 2,1\}$. Clearly $u=v=0$ is not an equilibrium strategy because the corresponding payoff (mean of $F$ ) is positive, so say Player $I$ should reject $x=0$, contrary to what the strategy specifies. For strategy $u=v=1 / 2$ all candidates but $x=y=0$ will be accepted, so the mean of say $x$ for hired candidates will be

$$
\frac{(p / 2)+q}{1-(1-p-q)^{2}}
$$

For strategy $u=v=1$ the payoff is $\frac{(p q) / 2+q}{2 q-q^{2}}$. So the equilibrium conditions corresponding to the two strategies are that

$$
\begin{aligned}
\frac{(p / 2)+q}{1-(1-p-q)^{2}} & <\frac{1}{2}, \text { for strategy } u=v=1 / 2 \\
\frac{(p q) / 2+q}{2 q-q^{2}} & >\frac{1}{2}, \text { for strategy } u=v=1 .
\end{aligned}
$$


The second condition is always satisfied (so $u=v=1$ is always an equilibrium), and the first is satisfied (so $u=v=1 / 2$ is an equilibrium) for

$$
q<\sqrt{p}-p
$$

the region below the curve in Figure 8. This example illustrates the possibility of multiple equilibria, although we shall rule this out in Theorem 17 for the uniform distribution.

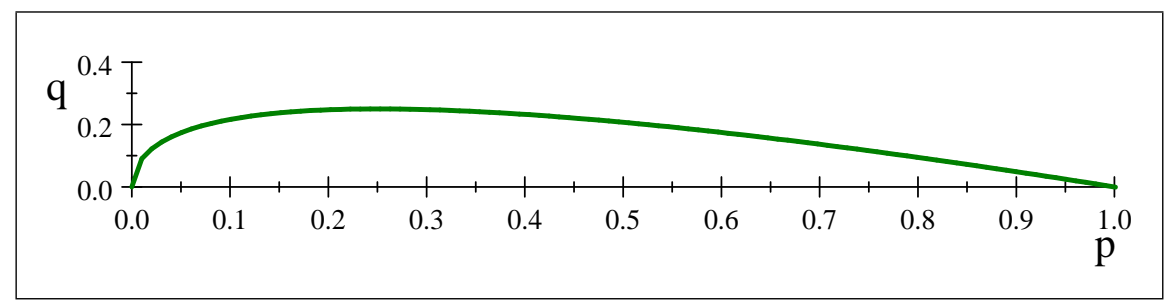

Figure 8. Two equilibria for, $q<\sqrt{p}-p$, otherwise just $u=v=1$.

\section{Existence of equilibria in $G(0,0)$ for i.i.d. distri- butions}

The reader will have noticed that for the i.i.d. distributions in our Examples 7, 10 and 6, where $F$ consists of two or three atoms, or is uniform, we always found at least one equilibrium. We now show that an equilibrium exists for any such distribution $F$. By Theorem 9 , we need consider only symmetric equilibria $u=v$, where I accepts $(x, y)$ if $x \geq u$ and II accepts $(x, y)$ if $y \geq u$. In this section let

$$
\Phi(u)=d T(u, u)
$$

denote $d$ times the expected payoff to either player when the symmetric strategy $(u, u)$ is adopted. Recall that any fixed point $u$ of $\Phi$ (solution of $u=\Phi(u)=d T(u, u))$ is an equilibrium. Note that $\Phi:[0,1] \rightarrow[0,1]$ is continuous except possibly at atoms of $F$, and at such atoms it is continuous from the left. If $F$ has no atoms, there is clearly a fixed point (Intermediate Value Theorem or Brouwer's Theorem in one dimension) as seen in Figure 9. We will show that a fixed point exists even when $\Phi$ has discontinuities. 
What we have to exclude is the situation drawn in Figure 9 , where $\Phi(u)$ has a discontinuity at $a$ where $\Phi(a)>a$, and $\Phi\left(a^{+}\right)=\lim _{p \backslash 0} \Phi(a+p)<a$. That is, $\Phi$ jumps from a point above the diagonal down to a level below the diagonal.

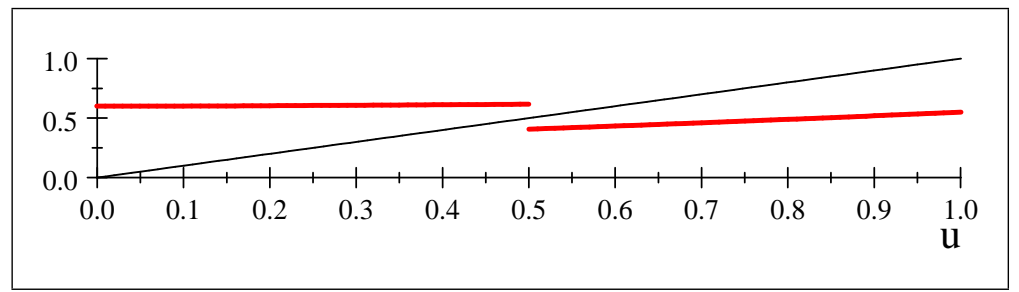

Figure 9. No fixed point due to discontinuities

So what property does the curve $\Phi(u)$ possess, for any distribution $F$, that avoids the behavior of the curve of Figure 12?. The answer is simple: jumps that occur starting above the diagonal (from $(a, \Phi(a)$ ) with $\Phi(a)>a$ ) are always 'up' $\left(\Phi\left(a^{+}\right)>\Phi(a)\right)$. We call this property correctly jumping, and base our proof on it.

Recall the definition of the hired set $H$,

$$
H=H(u, v)=\{(x, y): x \geq u \text { or } y \geq v\}
$$

and modify it for the symmetric case as

$$
H=H(u)=H(u, u)=\{(x, y): x \geq u \text { or } y \geq u\}
$$

We shall sometimes use the notation

$$
H\left(u^{+}\right)=\{(x, y): x>u \text { or } y>u\}
$$

to denote the hired candidates in the case that the players accept candidates only if they are strictly above their threshold level.

Definition 11 A real function $\Phi$ which is left continuous and satisfies $\Phi(a)>$ 
$a \Longrightarrow \Phi\left(a^{+}\right) \equiv \lim _{p \backslash 0} \Phi(a+p)>\Phi(a)$ at its discontinuities is called correctly jumping.

Lemma 12 The function $\Phi(u)=d T_{1}(u, u)$ for $G(0,0)$ with an i.i.d. distribution $F$ is correctly jumping.

Proof. Suppose that $\Phi(a)>a$ for some atom $a$ of $F$. Partition the hired set $H=H(a)$ into $H^{+} \equiv H\left(a^{+}\right)$and the difference set $D \equiv H(a)-H\left(a^{+}\right)$ (see $(17,18))$. The latter set $D$ is the union of the two line segments from $(a, a)$ to $(a, 0)$ and to $(0, a)$. Since $x \leq a$ on $D$, we have $E_{1}(D) \leq a$. By $(7)$, we have

$$
\begin{aligned}
\Phi(a) & =d T_{1}(a, a)=k E_{1}(H), \\
\text { where } k & =d \frac{\mu(H)}{1-(1-\mu(H)) d}=\frac{\mu(H)}{\mu(H)+(1 / d-1)} \leq 1
\end{aligned}
$$

Since

$$
a<\Phi(a)=k E_{1}(H(a))=\frac{\mu\left(H^{+}\right)}{\mu(H)} k E_{1}\left(H^{+}\right)+\frac{\mu(D)}{\mu(H)} k E_{1}(D)
$$

and $E_{1}(D) \leq a<\Phi(a)$, we must have $k E_{1}\left(H^{+}\right)>a$. But $\Phi\left(a^{+}\right)=$ $k E_{1}\left(H^{+}\right)>a$.

The alert reader will note that the idea in the proof establishes the stronger result that if $u<\Phi(u)$ then $\Phi$ is increasing on the interval $[u, \Phi(u)]$ so that in particular any jumps on this interval must be upwards. But we will not need that result. We can now modify the usual proof of the Intermediate Value Theorem to establish the following elementary fixed point result.

Theorem 13 Let $\Phi:[0,1] \rightarrow[0,1]$ be correctly jumping. Then for some $u \in[0,1]$, we have $\Phi(u)=u$.

Proof. We may assume that $\Phi(0)>0$ and define $a=\sup \{x: \Phi(x)>x\}$. Since $\Phi$ is continuous from the left, we have $\Phi(a) \geq a$. If $\Phi(a)=a$ we are done, so assume $\Phi(a)>a$. Since $\Phi$ is correctly jumping, we have $\Phi\left(a^{+}\right)>$ $\Phi(a)$. Consequently $\Phi\left(a^{+}\right)>\Phi(a)>a$. This implies that some $x>a$ 
satisfies $\Phi(x)>x$. This contradicts the definition of $a$, so our assumption that $\Phi(a)>a$ was false, and $\Phi(a)=a$.

Corollary 14 The game $G(0,0)$ has at least one equilibrium, for any symmetric candidate distribution and any discount factor $d<1$.

Proof. The expected payoff function $\Phi(u)$ for $G(0,0)$ is correctly jumping, by Lemma 9 . Hence by Theorem 10 there is a solution $u=d \Phi(u)$ for any discount factor $d$. The pair $(u, u)$ is therefore an equilibrium of $G(0,0)$.

\section{Behavioral Strategy Equilibria in $G(0,0)$}

In this section we show that the restriction to i.i.d. candidate distributions in Corollary 14 can be relaxed if we are willing to consider randomized strategies. Suppose we are looking for an equilibrium strategy pair with payoffs $(U, V)$. Consider Player I's strategy. At equilibrium, he must accept any candidate with $x>u=d U$ and he must reject any candidate with $x<d U$. He can accept a candidate with $x=u$ with any probability $p$. Denote such a behavioral strategy for I by $[u, p]$. Note that if the probability of a candidate with $x=u$ is zero, the strategies $[u, p]$ give the same payoff as the pure strategy $u$ (denoting accept iff $x \geq u$ ). Extend the definition of $T$ such that $T([u, p],[v, q])$ is the payoff pair corresponding to these mixed strategies. Then let

$$
T^{*}(u, v)=\cup_{p, q} T([u, p],[v, q])
$$

be the set of all payoff pairs corresponding to mixed strategies based on $u$ and $v$. Note that for any pair $(u, v), T^{*}(u, v)$ is a closed convex subset of $[0,1]^{2}$, and that $T^{*}$ has a closed graph. So it follows from Kakutani's fixed

point theorem that there exists a (fixed point) pair $(u, v) \in[0,1]^{2}$ such that

$$
(u, v) \in T^{*}(u, v)
$$


This means that for some probabilities $p$ and $q$, we have

$$
T([u, p],[v, q])=(u, v)
$$

which implies that the mixed strategy pair $([u, p],[v, q])$ is an equilibrium. Since we have made no assumption about the candidate distribution (which is implicit in the definition of $T$ ) we have established the following.

Theorem 15 For any candidate distribution, and any discount factor $d<$ 1 , there exists an equilibrium for $G(0,0)$ in behavioral strategies.

A similar argument involving randomizing at atoms of a population, in the context of two-sided matching, is given in Alpern and Reyniers (2005). If $d=1$ the graph of $T$ might not be a closed set. To see this, consider the uniform candidate distribution and take our usual assumption that $T(1,1)=(0,0)$. (We could be more formal and write $T([1,0],[1,0])=(0,0)$ to indicate that a candidate with type $x=1(y=1)$ will not be accepted by Player I (II) ). Then $(1-1 / n, 1-1 / n) \rightarrow(1,1)$ but $T^{*}(1-1 / n, 1-1 / n)=$ $T(1-1 / n, 1-1 / n)$ (equating a point with a singleton set) converges to $(3 / 4,3 / 4)$; and $(3 / 4,3 / 4) \notin T^{*}(1,1)=\{(0,0)\}$. Thus in this case $T$ does not have a closed graph. If there were a positive probability of $x=1$ or $y=1$ then this would not be a problem.

\section{Analysis of $G(i, j)$, Uniform Distribution}

In Example 6, we obtained the unique equilibrium for the no-veto game $G(0,0)$ when the distribution was uniform. We now extend that analysis to the case where the players have vetoes. We show how a unique progressive equilibrium of $G(i, j)$ can be obtained, given equilibria of its two immediate subgames, by iterating the expected value function $T$.

We seek an progressive equilibrium pair $(u, v)$ as described in Figure 3 for $G(i, j)$ which is supported by an equilibrium with payoffs $(a, e) \equiv\left(u_{-}, v_{+}\right)$in $G(i-1, j)$ and one with payoffs $(b, c)=\left(u_{+}, v_{-}\right)$in $G(i, j-1)$. According to $(5)$ any $(u, v)$ in the rectangle $R_{1}=[a, b] \times[c, e]$ satisfying the fixed point 
equation

$$
\begin{gathered}
(u, v)=d T(u, v), \text { with } \\
T_{1}=\frac{1}{2}\left[a^{2} v-c u^{2}+u^{2} v+2 a c u-2 a u v+\left(1-2 a^{2} c-c+2 c e+a^{2}\right)\right] \\
T_{2}=\frac{1}{2}\left[c^{2} u-a v^{2}+u v^{2}+2 a c v-2 c u v+\left(1-a+2 a e+c^{2}-2 a c e\right)\right],
\end{gathered}
$$

is a progressive equilibrium. It is easy to see that $T$ maps the larger rectangle $R_{2}=[a, 1] \times[c, 1]$ into itself, as all outcomes of $G(i, j)$ (hired candidates or values of subgames) when the strategy $(u, v)$ is adopted have payoffs in this rectangle, and hence so do averages of these, since $R_{2}$ is convex.

Lemma 16 For any constants $1 / 2 \leq a \leq b \leq 1,1 / 2 \leq c \leq e \leq 1$, the map $T: R_{2} \rightarrow R_{2}$ is a contraction map.

Proof. The Jacobian of $T(u, v)$ is given by

$$
J=\frac{1}{2}\left(\begin{array}{cc}
2(u-a)(v-c) & (u-a)^{2} \\
(v-c)^{2} & 2(u-a)(v-c)
\end{array}\right) .
$$

To compute $\|J\|$, we note that eigenvalues are equal to $\frac{1}{2}(v-c)(u-a)$ and $\frac{3}{2}(v-c)(u-a)$. Thus the value of the larger eigenvalue gives

$$
\|J\|=\frac{3}{2}(v-c)(u-a) \leq \frac{3}{2}(1-c)(1-a) \leq \frac{3}{4}<1, \text { for }(u, v) \in R_{2} .
$$

Theorem 17 (Uniqueness) Any pair of equilibria for the subgames $G(i-1, j)$ and $G(i, j-1)$ with respective values $(a, e),(b, c), 1 / 2 \leq a \leq b \leq 1$, $1 / 2 \leq c \leq e \leq 1$, supports at most one progressive equilibrium $[u, v]_{\text {prog }}$ for the game $G(i, j)$. Furthermore, any such equilibrium can be obtained as the limit of $T^{n}$, starting at any point in $R_{2}$. The same result holds with any discount factor $d$.

Proof. Since $T$ is a contraction on the rectangle $R_{2}$, the Contraction Mapping Theorem implies that it has exactly one fixed point in $R_{2}$ and hence at 
most one fixed point in the subrectangle $R_{1}$ (which would be a progressive equilibrium). Furthermore we know that the fixed point is attracting from any point in $R_{2}$. If there is a discount factor $d<1$ the same proof holds for the mapping $d T$, with Jacobian norm $\frac{3 d}{2}(v-c)(u-a)$.

The restriction to supporting equilibria with values at least $1 / 2$ is not a problem, as all progressive equilibria clearly have values greater than $1 / 2$. We can now use Theorem 17 to obtain equilibria by iterating $T$ for fixed $d$ in successive games $G_{d}(i, j)$, as long as we do so in a manner that deals with $G(i-1, j)$ and $G(i, j-1)$ before $G(i, j)$. If the iteration converges to a point in $R_{1}$ (as it always has done in our computations), we know that this equilibrium is the unique one. Equilibria obtained recursively in this fashion will of course be strongly progressive, since all the supporting equilibria will be progressive. Note that our analysis does not rule out the possibility that the iterative process converges to a point of $R_{2}-R_{1}$, a fixed point which would not correspond to a progressive equilibrium. We give below the results of our Mathematica calculations for the unique equilibria $(u, v)$ for the undiscounted games $G(i, j), i, j=0, \ldots, 4$, for the uniform distribution. Note the already calculated values $u=v=.618034$ (golden mean) for $G(0,0)$, the symmetry between $G(i, 0)$ and $G(0, i)$, and the lack of symmetry otherwise between $G(i, j)$ and $G(j, i)$.

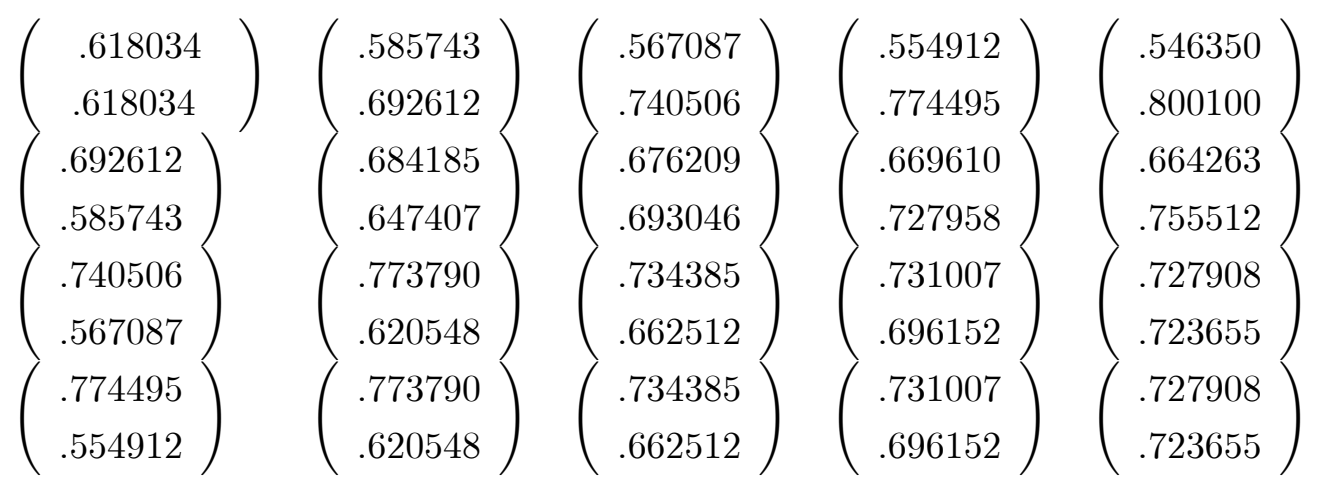

Table 1. Equilibrium values for $G(i, j), d=1$.

Note that the first coordinate $u$ increases as we go down (the number $i$ of first player vetoes increases), and similarly $v$ increases as we move to the 
right. That is, $u>u_{-}$and $v>v_{-}$. Also, the equilibria are progressive in that $u<u_{+}$(the first coordinate decreases as we move to the right) and similarly $v<v_{+}$. That is, giving an extra veto to the other player hurts you. However when the future is discounted $(d<1)$ things can be more complicated.

$$
\begin{array}{lll}
(0.5541,0.5541) & (0.4920,0.6125) & (0.4607,0.6381) \\
(0.6125,0.4920) & (0.5630,0.5444) & (0.5344,0.5706) \\
(0.6381,0.4607) & (0.5901,0.5113) & (0.5624,0.5376)
\end{array}
$$

Table 2. Equilibrium values for $d=.86$

For example, when $d=.86$, both players do better (on average) when both have a single veto than when both have two vetoes. Note that a firm which values $x$ and $y$ equally does best when neither player has a veto. This last consideration will be studied in the next section.

\section{Optimal Design of Committees}

In this section we view the problem of committee design from the point of view of the firm. We assume the firm has some utility function $U(x, y)$ for hiring a candidate $(x, y)$. It is reasonable to assume that $U$ is increasing in $x$ and $y$, for otherwise why would it put players I and II (who value $x$ and $y$ positively) on the hiring committee in the first place. For simplicity, we will take the firm's utility function as $x+y$, though the analysis we do here can obviously be done for any function $U$. First consider the case of no discounting, $d=1$. If we add the coordinates in Table 1 , we see that the firm's utility is increasing in both $i$ and $j$, and it should give as many vetoes 
to the committee as possible.

$\begin{array}{ccccc}1.23607 & 1.27835 & 1.30759 & 1.32941 & 1.34645 \\ 1.27835 & 1.33159 & 1.36925 & 1.32941 & 1.34645 \\ 1.30759 & 1.33159 & 1.39690 & 1.42716 & 1.41977 \\ 1.32941 & 1.37597 & 1.41301 & 1.44313 & 1.46807 \\ 1.34645 & 1.38906 & 1.42399 & 1.45311 & 1.47776 \\ \text { Table 3. Sum of values in Table } 1, \mathrm{~d}=1\end{array}$

However the firm has an interest in hiring candidates quickly, which we assume involves the same discount factor as the player (though this is not required for our analysis). The more the future is discounted, the fewer vetoes it should give the committee members (players I and II). If $d$ is less than .86 , the incentive to hire quickly results in giving no vetoes to either committee member. If $d=.87$, it is best to give the players one veto each.As the future is discounted less, more vetoes can be given, allowing better candidates to be hired.

$\begin{array}{lllllllll}1.1195 & 1.1206 & 1.1174 & 1.1134 & 1.1393 & 1.1485 & 1.1499 & 1.1483 & 1.1460 \\ 1.1206 & 1.1286 & \mathbf{1 . 1 2 8 7} & 1.1263 & 1.1485 & 1.1649 & 1.1702 & 1.1708 & 1.1696 \\ 1.1174 & 1.1244 & 1.1255 & 1.1244 & 1.1499 & 1.1647 & 1.1707 & \mathbf{1 . 1 7 2 4} & 1.1722 \\ 1.1134 & 1.1197 & 1.1213 & 1.1209 & 1.1483 & 1.1616 & 1.1677 & 1.1699 & 1.1704\end{array}$

Table 5.Optimal numbers of vetos, $d=.88$ (left) and $d=.91$ (right).

It appears from our numerical results that it is optimal to give the players roughly equal numbers of vetoes, with the weaker Player II (who moves last) at least as many as the stronger Player I.

\section{Conclusions}

This paper models the process of sequentially selecting a candidate for a position as an alternating-move perfect-information game. Strong negative evaluations of a candidate by a committee member are reflected by allowing 
the member to veto such a candidate, but at the cost of fewer remaining vetos. We analyze this game, in particular showing that if candidates are uniformly distributed in their valuations to committee members, then there is a unique equilibrium corresponding to each discount factor. The equilibrium depends crucially on the number of vetos initially assigned to each committee member. Knowing the effect of this veto distribution, the firm can optimize it to obtain the best candidates, from it's point of view.

The game $G(i, j)$ that we define in this paper has many aspects still to be understood for other or arbitrary distributions: existence questions for equilibria, the 'donation paradox' (wanting to give a veto to the other member), simultaneous moves (closed ballots). A second more technical paper is planned to deal with these aspects of the game. In particular, we believe more general existence results may be obtained by using the theory of abstract stopping games, as in Shmaya and Solan, S. (2004). It should be noted that if players know only their own valuations of candidates then the analysis of the resulting game is much simpler, as strategies are functions of a single variable; the complexities of our game result from the knowledge of others' valuations.

\section{Bibliography}

S. Alpern and D. Reyniers (2005). Strategic mating with common preferences. Journal of Theoretical Biology 237, no. 4, 337-354.

V. Baston and A. Garnaev (2007). Competition for staff between two departments. Game Theory and Applications 10, in press.

S. J. Brams and M. D. Davis (1976). A game-theory approach to jury selection. Trial 12, 47-49.

S. J. Brams and M. D. Davis (1978). Optimal jury selection: A Game theoretic model for the exercise of peremptory challenges. Operations Research 26, 966-991. 
M. H. DeGroot and J. B. Kadane (1980). Optimal challenges for selection. Operations Research 28, no. 4, 952-968

Eriksson, K., Sjöstrand, J. and Strimling, P. (2007). Optimal expected rank in a two-sided secretary problem, Operations Research, in press.

Ferguson, T. (1989). Who solved the secretary problem? Statist. Sci. 4, 282-296.

Ferguson, T. (2005). Selection by Committee. Annals International Society of Dynamic Games 7, 203-210.

Roth, A., and J. B. Kadane and M. H. DeGroot (1977). Optimal peremptory challenges in trials by juries: A bilateral sequential process. Operations Research 25, 901-919.

Shmaya, E. and Solan, S. (2004). Two-player non zero-sum stopping games in discrete time. Annals of Probability 32, 2733-2764. 